\section{Forensic use of PCR in Italy}

SIR - Following the admission of the use of evidence obtained through the polymerase chain reaction (PCR) in British courts ${ }^{1}$, attention has been drawn to the forensic use of PCR in Italy. Dallapiccola et al. ${ }^{2}$ reported that at the Balsorano-Avezzano court case they were able irrefutably to involve a man in a murder by a PCR-driven DNA analysis, providing arguments in favour of PCR use and a reliable protocol for routine work.

Having acted as expert witnesses at that trial, we believe that the evidence produced was of dubious value and the protocol deserved severe criticisms.

(1) Although they recommended great care in producing forensic evidence by DNA profiles, Dallapiccola et al. carried out a technically flawed analysis. Overlooking elementary rules to assert a match between forensic samples, they type amplified VNTRs (variable number of tandem repeats; for example D1S80) and hypervariable restriction fragment length polymorphisms (D2S44) - endowed with quasi-continuous variability - on small-size electrophoresis with inadequate resolution, rough molecular mass ladders and no allelic standards. Having no population database for their markets, they could not draw any warranted probability estimate. Being ignorant of the precautions to adopt in referring statistical inferences to substructured populations ${ }^{3,4}$, they overlooked the fact that the Appennine mountain community where the crime was committed (Borgo Case Castella di Balsorano, 97 inhabitants in 1990) is in fact a genetic isolate with a high rate of inbreeding. Against this background, they issued an arbitrary probability figure $\left(1: 6 \times 10^{-4}\right)$, which nonetheless influenced the jury. In short, they fell into just the situation of 'worthless evidence' and 'unreliable conclusions' against which they warn.

(2) We are very surprised to find that in their letter ${ }^{2}$ the impact of DNA analysis in this court case is even more exaggerated than in their original, written report at the trial, as they now quote hyperbolic, seven orders of magnitude higher odds $\left(1: 1.7 \times 10^{-11}\right)$ to indict the defendant.

(3) Last but not least, the protocol they used is not suitable for forensic analysis. Largely based on scarcely polymorphic systems, not validated for forensic purposes (APO-CII, 3'CACA, distrophin, VNDR18 and others), it lacks standardization, quality controls and mutual objectivity. By disregarding so many obligatory issues to produce scientific evidence in $\operatorname{court}^{5}$, Dallapiccola et al. rendered the wrong service to a good cause (forensic use of PCR). As part of a group promoting standardization and quality assurance in the field (the European DNA profiling group, EDNAP) we denounce this example of redundant, as well as unsuitable forensic analysis.

ANGELO FIORI

VINCENZO L. PASCALI

Department of Forensic Medicine,

Catholic University of Sacred Heart,

largo F. Vito, 1 ,

00168 Roma, Italy

1. Hagelberg, E., Gray, I. C. \& Jeffreys, A. J. Nature 352 , 427-429 (1991)

2. Dallapiccola, B., Novelli, G. \& Spinella, A. Nature 354, 179 (1991).

3. Lewontin, R. C. \& HartI, D. L. Science 254, 1745-1750 (1991)

4. Brookfield, J. Nature 355, 207-208 (1992)

5. Nature 350, 95 (1991)

\section{Air pollution and cancer risk}

SIR - It is pleasing to learn from Paolo Paoletti et al. (Nature 355, 290; 1992) that serious efforts are under way within at least one country of the European Communities (Italy) to determine the effects of air pollution upon health, including cancer risk.

The British view is that the cancer risk to the general population from vehiclederived air pollution is so small as to be unmeasurable (F. Godlee $\mathrm{Br}$. med. $J$. 303, 1539-1543; 1991). Lung cancer in Britain is now attributed almost solely to active and passive smoking (with, perhaps, a small contribution from domestic radon). The million-odd tonnes of known or probable carcinogen churned out into the air by road transport annually is deemed innocuous.

Yet observations that seem to conflict with this view (urban are higher than rural lung cancer rates, risk is highest in the most densely trafficked urban areas and so on) are dismissed on the basis of local differences in socioeconomic factors or smoking behaviour. But it is difficult to explain why lung cancer rates in rural China are an order of magnitude lower than in Britain, and not much different between smokers and nonsmokers ${ }^{1}$.

Interestingly, when the increase in lung cancer rate in developed countries was first noticed earlier this century, it was a moot point whether air pollution (particularly from the new-fangled diesel lorries and buses) or tobacco smoking was to blame. That the entire blame has been laid at the door of smoking alone has been a source of mystery to medical sociologists ${ }^{2}$. The likely possibility of synergy between powerful mutagenic factors adsorbed on diesel particulates and cancer-promoting factors in cigarette smoke has never been closely assessed and, over the decades, any possible contribution of air pollution to lung cancer has been carefully edited out, perhaps, I have suggested ${ }^{3}$, to the quiet but forceful involvement of groups outside medical science with a natural interest in vehicular air pollution proving to be harmless.

Wide-ranging prospective surveys such as those proposed by Paoletti and his colleagues may indeed answer the question: "Does air pollution cause cancer?", but we also need cross-sectional surveys of risk factors in patients. As air pollution caused by traffic tends not to be homogeneous, it should be feasible not only to ask lung cancer patients "How many cigarettes do you smoke each day?" but to ask local traffic engineers how many vehicles pass their residence.

SIMON P. WolfF

Department of Clinical Pharmacology,

UCMSM,

University College London,

5 University Street

(The Rayne Institute),

London WC1E 6J, UK 1. Katz, R. \& Chunxiang, Z. Hith. Phys. 51, $457-468$
(1986).
2. Vandenbroucke, J. P. Am. J. Epidemiol. 133, $426-427$
(1991).
3. Wolff, S. P. Am. J. Epidemiol. (in the press).

\section{Authors and egos}

SIR - Christopher Anderson's report (Nature 355, 101; 1992) on publication frequency by scientists should be cause for grave concern in the scientific community. Authorship of a scientific publication is not a reward for having assisted in some way, however trivial, in making a research report possible.

If one is conducting field research in the Andes, for example, the muleteer hired to provide access to the study area obviously makes a vital contribution to the research effort but few would argue that this effort warrants co-authorship of any publications resulting from the expedition's scientists. Similarly, someone who is awarded a grant does not automatically merit co-authorship merely because the funds made possible the research of others, any more than the largesse of an individual private donor who provides funds for research would merit inclusion on an author line.

If this were not a serious problem in science, the almost childlike attempts to feed enormous egos would be silly, indeed. Does anyone really believe that someone authors a paper every few days? Clearly not. Perhaps it is time for reviewers of manuscripts and grants to stop playing this little game and deduct points for patent ego engorgement.

Michael A. MARES

Oklahoma Museum of Natural History

and Department of Zoology,

University of Oklahoma,

Norman, Oklahoma 73019, USA 